\title{
Protease Activated Receptor 2 (PAR2) Induces Long-Term Depression in the Hippocampus through Transient Receptor Potential Vanilloid 4 (TRPV4)
}

\author{
Efrat Shavit-Stein 1, Avital Artan-Furman', Ekaterina Feingold1, Marina Ben Shimon 1,2, \\ Zeev Itzekson-Hayosh', Joab Chapman ${ }^{1,2,3}$, Andreas Vlachos ${ }^{4}$ and Nicola Maggio 1,2,3,5* \\ 'Department of Neurology, The Chaim Sheba Medical Center, Tel HaShomer, Israel, ${ }^{2}$ Department of Neurology and \\ Neurosurgery, Sackler Faculty of Medicine, Tel Aviv University, Tel Aviv, Israel, ${ }^{3}$ Sagol School of Neuroscience, Tel Aviv \\ University, Tel Aviv, Israel, ${ }^{4}$ Institute of Anatomy II, Faculty of Medicine, Heinrich-Heine-University, Duesseldorf, Germany, \\ ${ }^{5}$ Talpiot Medical Leadership Program, The Chaim Sheba Medical Center, Tel HaShomer, Israel
}

OPEN ACCESS

Edited by:

Christian Alzheimer, University of Erlangen-Nuremberg,

Germany

Reviewed by: Thomas Mittmann,

University of Mainz, Germany Daniel John Whitcomb,

University of Bristol, UK

${ }^{*}$ Correspondence: Nicola Maggio nicola.maggio@sheba.health.gov.il

Received: 19 October 2016 Accepted: 07 February 2017 Published: 02 March 2017

Citation:

Shavit-Stein E, Artan-Furman A, Feingold E, Ben Shimon M, Itzekson-Hayosh Z, Chapman J, Vlachos A and Maggio N (2017) Protease Activated Receptor 2 (PAR2) Induces Long-Term Depression in the Hippocampus through Transient Receptor Potential Vanilloid 4 (TRPV4). Front. Mol. Neurosci. 10:42. doi: 10.3389/fnmol.2017.00042
Protease activated receptors (PARs) are involved in regulating synaptic transmission and plasticity in the brain. While it is well-accepted that PAR1 mediates long-term potentiation (LTP) of excitatory synaptic strength, the role of PAR2 in synaptic plasticity remains not well-understood. In this study, we assessed the role of PAR2-signaling in plasticity at hippocampal Schaffer collateral-CA1 synapses. Using field potential recordings, we report that PAR2-activation leads to long-term depression (LTD) of synaptic transmission through a protein kinase A -dependent, Transient Receptor Potential Vanilloid 4 -mediated mechanism, which requires the activation of $\mathrm{N}$-methylD-aspartate receptors. These results demonstrate that the effects of PAR2 on synaptic plasticity are distinct from what is observed upon PAR1-activation. Thus, we propose that the activation of different classes of PARs, i.e., PAR1 and PAR2, may set the threshold of synaptic plasticity in the hippocampal network by balancing LTP and LTD.

Keywords: PAR2, TRPV4, synaptic plasticity, hippocampus, LTD

\section{INTRODUCTION}

Synaptic plasticity in the hippocampus underlies the formation of certain forms of memory, including spatial memory (Malenka, 2003; Maggio and Vlachos, 2014). A number of molecules have been postulated to be involved in long-term potentiation (LTP) with recent studies reporting a fundamental role of extracellular proteases in this process (Wlodarczyk et al., 2011; Tsilibary et al., 2014). Among others, protease activated receptors (PAR), which are activated by serine proteases, have been shown to modulate neural excitability and synaptic plasticity (Almonte et al., 2007, 2013; Traynelis and Trejo, 2007; Ben Shimon et al., 2015). PARs belong to a unique family of $G$ protein-coupled receptors (Luo et al., 2007). Their activation is initiated by an irreversible, site-specific proteolytic cleavage in the $\mathrm{N}$-terminal extracellular region, which uncovers a tethered ligand activating $\mathrm{G} \alpha_{\mathrm{q} / 11}, \mathrm{G} \alpha_{\mathrm{i} / \mathrm{o}}$, or $\mathrm{G} \alpha_{12 / 13}$-proteins (Coughlin, 2001; Macfarlane et al., 2001; Traynelis and Trejo, 2007). While PAR1-activation has been shown to modulate synaptic transmission and plasticity through the enhancement of $\mathrm{N}$-methyl-D-aspartate receptor (NMDAR) currents (Gingrich et al., 2000; Lee et al., 2007; Maggio et al., 2008; Becker et al., 2014; Vance et al., 2015), and PAR1-deficient animals show 
alterations in hippocampus-dependent learning and memory processes (Almonte et al., 2007, 2013), the role of PAR2 in neural function and plasticity remains not well-understood.

Unlike other members of the PAR-family, which are effectively activated by thrombin, PAR2 represents a class of trypsin/tryptase-activated receptors (Nieman, 2016). Among other PAR2-activators are tissue kallikreins, coagulation factors VIIa and Xa, and transmembrane serine proteases (Oikonomopoulou et al., 2006; Rezaie, 2014). PAR2 is involved in mediating important biological functions such as inflammation, coagulation, and immunity (Rothmeier and Ruf, 2012; Rezaie, 2014; Bushell et al., 2016). Although PAR2 is detected in the brain (Bushell et al., 2006; Luo et al., 2007; Olianas et al., 2007), and recent work has indicated a role for PAR2 in synaptic plasticity (Lohman et al., 2009; Gan et al., 2011), the molecular signals through which PAR2 affects synaptic transmission and plasticity in the CNS remain unknown.

In the peripheral nervous system PAR2-activation has been linked to neuroinflammation and neuropathic pain (Noorbakhsh et al., 2006; Bao et al., 2014; Tillu et al., 2015; Bushell et al., 2016), through a protein kinase A (PKA)-dependent activation of Transient Receptor Potential Vanilloid 4 (TRPV4) channels (Grant et al., 2007; Chen et al., 2011; Poole et al., 2013). TRPV channels belong to a family of non-selective cation channels that are activated by a wide variety of chemical and physical stimuli (Gunthorpe et al., 2002). While they are highly expressed in sensory neurons in the peripheral nervous system, a number of studies have also reported TRPV expression in the brain (Alter and Gereau, 2008; Shibasaki et al., 2015), though their functions are less well-understood. Recent evidences point toward a role of TRPV channels in hippocampal synaptic plasticity (Gibson et al., 2008; Edwards et al., 2012; Brown et al., 2013). Therefore, we sought to address the hypothesis that PAR2-activation affects synaptic plasticity through TRPV4.

\section{MATERIALS AND METHODS}

\section{Chemicals}

The following compounds were used at the following concentrations: $10 \mu \mathrm{M}$ AC55541 (PAR2-agonist, Tocris Bioscience, $\mathrm{UK}$ ), $10 \mu \mathrm{M}$ AC264613 (PAR2-agonist, Tocris Bioscience, UK), $50 \mu \mathrm{M}$ FSLLRY-NH ${ }_{2}$ (PAR2-antagonist, Sigma-Aldrich, Israel), $2 \mu \mathrm{M}$ RN1747 (TRPV4-agonist, Tocris Bioscience, UK), $10 \mu \mathrm{M}$ RN1734 (TRPV4-antagonist, Tocris Bioscience, UK), $10 \mu \mathrm{M}$ RN9893 (TRPV4-antagonist, Tocris Bioscience, UK), $50 \mu \mathrm{M}$ D(-)-2-amino-5-phosphonovaleric acid (APV, NMDARantagonist, Sigma-Aldrich, Israel), $200 \mu \mathrm{M}$ ( \pm -a-Methyl(4-carboxyphenyl)glycine (MCPG, mGluR-antagonist, SigmaAldrich, Israel), KT5720 (protein kinase A inhibitor, Tocris Bioscience, UK), GF109203x (protein kinase C inhibitor, Tocris Bioscience, UK). Pharmaceuticals were added to the perfusion medium with special care to prevent changes in temperature, $\mathrm{pH}$, flow rate, or degree of oxygenation of the artificial CSF (aCSF). Handling and disposal of all drugs carried out in accordance to National and Institutional regulations.

\section{Electrophysiology}

This study and protocol was approved by the Sheba Medical Center Institutional Animal Care and Use Committee (1000/15), which adheres to the national law, and NIH rules. Briefly, 45 months old male C57BL/6 mice were rapidly decapitated and $350 \mu \mathrm{m}$ coronal slices containing the dorsal hippocampus were used. Slices were incubated for $1.5 \mathrm{~h}$ in a humidified, carbogenated $\left(5 \% \mathrm{CO}_{2}\right.$ and $\left.95 \% \mathrm{O}_{2}\right)$ gas atmosphere at $33 \pm 1{ }^{\circ} \mathrm{C}$ and were perfused with a CSF [containing (in $\mathrm{mM}$ ) $124 \mathrm{NaCl}$, $2 \mathrm{KCl}, 26 \mathrm{NaHCO}_{3}, 1.24 \mathrm{KH}_{2} \mathrm{PO}_{4}, 2.5 \mathrm{CaCl}_{2}, 2 \mathrm{MgSO}_{4}$, and 10 glucose, $\mathrm{pH}$ 7.4] in a standard interface chamber. Recordings were made with a glass pipette containing $0.75 \mathrm{M} \mathrm{NaCl}(4 \mathrm{M} \Omega$ ) placed in the stratum radiatum CA1. A cut between CA3 and CA1 was made in order to avoid possible excitability. Stimulation was evoked using a Master 8 pulse stimulator (A.M.P.I., Jerusalem, Israel) and was delivered through two sets of bipolar nichrome electrodes placed on either side of the recording electrode such that two independent stimulation channels were used for each slice. The use of two parallel pathways allowed comparison of the effects of different drug application in the same slice (Maggio and Segal, 2007a,b). Longterm depression (LTD) was induced by low frequency stimulation (LFS) consisting of $1 \mathrm{~Hz}, 900$ pulses, as previously described (Maggio and Segal, 2009). Before applying the protocol, baseline values were recorded at a frequency of $0.033 \mathrm{~Hz}$. Responses were digitized at $5 \mathrm{kHz}$ and stored on a computer. Off-line analysis and data acquisition were performed using Spike 2 software (CED, Cambridge, England). All numerical data are expressed as mean \pm SEM, and EPSP slope changes after stimulation were calculated with respect to baseline. There were no systematic differences in the magnitudes of the baseline responses in the different conditions. Unless otherwise indicated, statistical evaluations were performed by applying Student's $t$-test for paired and unpaired data, as the case may be (Origin 8.0). $p$-values of $<0.05$ were considered a significant difference between means.

\section{Immunohistochemistry}

The following primary antibodies were used for immunodetection: goat anti-PAR2 (sc-8205, Santa Cruz, 1:25), rabbit anti-TRPV4 (ACC-124, Alomone Labs, 1:50), rabbit anti-PAR2 (APR-032, Alomone Labs 1:500) and mouse antiGFAP (G3893, Sigma-Aldrich, 1:2000). Hippocampal sections $(50 \mu \mathrm{m})$ were blocked in $10 \%$ normal horse serum in $0.1 \mathrm{M}$ $\mathrm{PBS} / 0.1 \%$ Triton for $1 \mathrm{~h}$ at room temperature (RT). After 24-48 h incubation at $4^{\circ} \mathrm{C}$ with the primary antibody (together with $2 \%$ normal horse serum), sections were exposed to the appropriate secondary antibody (DyLight ${ }^{\mathrm{TM}} 488$ conjugated affinity purified donkey anti-goat IgG, 1:800; Alexa Fluor 594 AffiniPure donkey anti-rabbit IgG, 1:2000; Alexa Fluor 488 conjugated AffiniPure donkey anti-mouse IgG, 1:400) for $1 \mathrm{~h}$. The sections were then washed, incubated with Hoechst (b1155, Sigma-Aldrich, $1 \mu \mathrm{g} / \mathrm{ml}$ final concentration) for $10 \mathrm{~min}$ (to allow nuclear staining), mounted on dry gelatin-coated slides and finally mounted and cover slipped with Flouromount (F4680, Sigma-Aldrich). Slides were imaged with a Leica SP5 confocal microscope and data were 
acquired and analyzed using a computer assisted image analysis system.

\section{RESULTS}

\section{PAR2-Activation Induces LTD at Schaffer Collateral-CA1 Synapses}

To test for the role of PAR2 in synaptic transmission and plasticity, we first treated acute hippocampal slices with the selective PAR2-agonist AC55541 $(10 \mu \mathrm{M})$ while recording evoked field potentials of Schaffer collateral-CA1 synapses. A profound depression of synaptic transmission was observed in these experiments reaching $0.73 \pm 0.07$ of baseline $30 \mathrm{~min}$ after bath-application of AC55541 ( $p<0.001 ; n=12$ slices, Figure 1A). Removal of the PAR2-agonist following induction of LTD did not affect the stability of synaptic depression (Figure 1B). To confirm the specificity of the PAR2-agonist, we repeated experiments in presence of the selective PAR2antagonist FSLLRY-NH 2 (50 $\mu \mathrm{M}$; washed in $15 \mathrm{~min}$ before $10 \mu \mathrm{M}$ AC55541). Indeed, in this experimental setting PAR2-LTD was not observed (Figure 1C). Similarly, activation of PAR2 using a different, specific agonist, i.e., AC264613 (10 $\mu \mathrm{M})$ also resulted in LTD and this effect was blocked by application of the PAR2antagonist (Supplementary Figures S1A,B, respectively). The effect of the PAR2-agonist (AC55541) was not concentrationdependent, since LTD of similar effects-size was observed when the PAR2-agonist was applied at concentrations of $0.1,1$, and $100 \mu \mathrm{M}$ (Figure 1D). We conclude from these experiments that PAR2-activation induces robust LTD at Schaffer collateral-CA1 synapses.

We then compared the dynamics of PAR2-LTD with LFSinduced LTD. In a two pathway experimental setting, the delivery of a $1 \mathrm{~Hz}$ protocol (900 pulses) resulted in a depression of $0.67 \pm 0.06$ at $30 \mathrm{~min}$, while the PAR2-agonist induced LTD of similar effect-size at the other pathway $(0.69 \pm 0.07, p=0.378$, $n=12$ ), without affecting the established LFS-LTD (Figure 1E). Moreover, the PAR2-antagonist did not affect the induction and maintenance of LFS-LTD, while preventing PAR2-LTD at the other pathway (Figure 1F). However, both forms of LTD required the activation of NMDAR, since $50 \mu \mathrm{M}$ of the NMDARantagonist APV blocked LFS-LTD and PAR2-LTD (Figure 1G).

Finally, we tested whether PAR2-mediated LTD is mGluRdependent by carrying out experiments in presence of the selective mGluR-inhibitor MCPG $(200 \mu \mathrm{M})$. Consistent with the literature (Maggio and Segal, 2007b; Fitzjohn et al., 2016), LFSLTD was partially impaired in these experiments $(0.82 \pm 0.06$, $p<0.01, n=12$, Figure $\mathbf{1 H})$. Yet, the induction of PAR2LTD was not affected by MCPG. Based on these results we conclude that PAR2-LTD requires the activation of NMDAR but not mGluR.

\section{TRPV4-Activation Induces LTD at Schaffer Collateral-CA1 Synapses}

PAR2 is known to mediate its effects, i.e., neuroinflammation and pain in the peripheral nervous system, through the activation of
TRPV4 channels (Grant et al., 2007; Chen et al., 2011; Poole et al., 2013). We therefore hypothesized that PAR2 may act on synaptic transmission via TRPV4.

To test this hypothesis, we first examined PAR2 and TRPV4 expression in the hippocampus. Anatomically matched frontal slices containing the dorsal hippocampus were immunostained for PAR2 and TRPV4. Indeed, both PAR2 and TRPV4 were expressed in the hippocampus. A comparable expression pattern was observed: high levels of PAR2 and TRPV4 were detected in CA1 stratum pyramidale. We did not find a prominent colocalization of PAR2 and the astrocytic marker GFAP in these experiments (Figure 2).

We then speculated that TRPV4-activation should also result in LTD, similar to what is observed upon PAR2-activation (c.f. Figure 1). This prediction was tested by exposing acute hippocampal slices to the TRPV4-agonist RN1747 (2 $\mu \mathrm{M})$. Indeed, a depression in synaptic transmission occurred, reaching $0.63 \pm 0.06 \%$ of baseline within $30 \mathrm{~min}(n=12$; Figure $3 \mathrm{~A}$; c.f. Figure 1A). This effect was long lasting as it persisted upon the removal of the TRPV4-agonist (Figure 3B). TRPV4-LTD was blocked in the presence of the TRPV4-antagonists RN1734 (10 $\mu \mathrm{M}$; Figure 3C) or RN9893, respectively (Supplementary Figure S1C). In two pathway experiments TRPV4-LTD reached similar levels of depression as compared to LFS-LTD $(0.66 \pm 0.07$ versus $0.72 \pm 0.05$ respectively, $n=12, p=0.19$, Figure $3 \mathrm{D}$ ), while LFS-LTD was not affected by the TRPV4-antagonist (Figure 3E). These experiments disclosed that TRPV4-activation induces robust LTD, similar to what is observed upon PAR2activation.

\section{PAR2 Induces LTD through the Activation of TRPV4}

In order to test for the interrelation between PAR2- and TRPV4-mediated LTD the following series of experiments was carried out. First, we exposed hippocampal slices to the TRPV4-antagonist before washing in the PAR2-activator (Figure 3F). Indeed, PAR2-activation with AC55541 (10 $\mu \mathrm{M})$ was not able to induce LTD in presence of the TRPV4-inhibitor RN1734 (10 $\mu \mathrm{M})$. Conversely, treatment with the TRPV4agonist in presence of the PAR2-antagonist reliably induced LTD $(0.64 \pm 0.07 \%$ of baseline after $30 \mathrm{~min}, n=12$; Figure $3 \mathrm{G})$.

We then examined whether sequential PAR2- and TRPV4activation occlude each other (Figure $3 \mathbf{H}$ ). Upon induction of PAR2-LTD, the PAR2-agonist was removed before washing in the TRPV4-agonist. In these experiments one of the two pathways was manually reported to the baseline value before TRPV4activation. Indeed, the TRPV4-agonist failed to induce LTD in this setting, suggesting that the pathway was already saturated, i.e., occluded by the prior application of the PAR2-agonist (Figure 3H).

Finally, we tested whether PAR2- and TRPV4-activation share the same molecular cascade requiring NMDAR but not mGluR activity (c.f. Figures 1G,H). Indeed, we observed that TRPV4activation did not induce LTD in presence of $50 \mu \mathrm{M}$ APV $(n=12$, Figure 4A), and similar to PAR2-LTD the mGluR-antagonist MCPG $(200 \mu \mathrm{M})$ was not effective in blocking TRPV4-LTD $(0.65 \pm 0.04, n=12$, Figure 4B). 
A

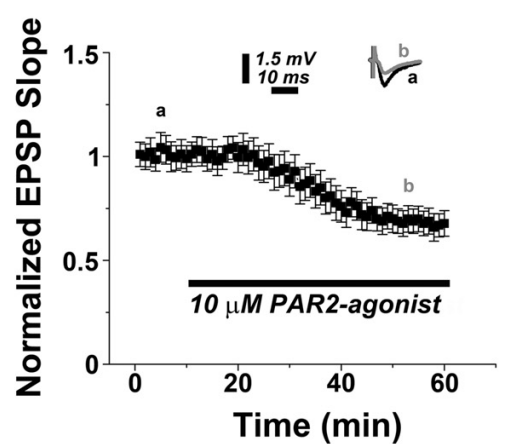

C

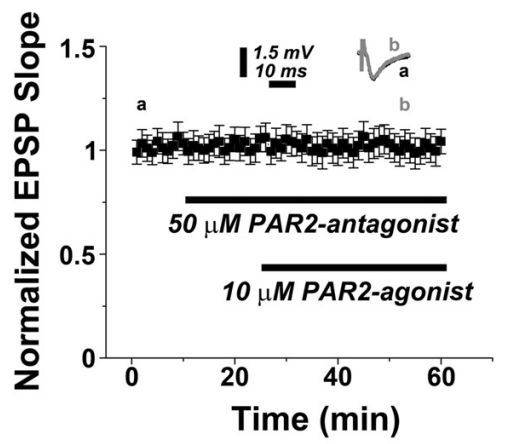

E

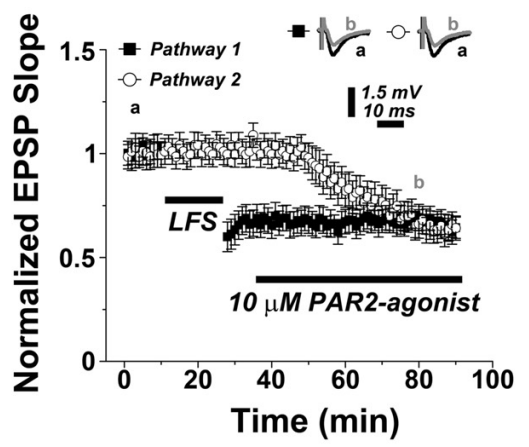

G

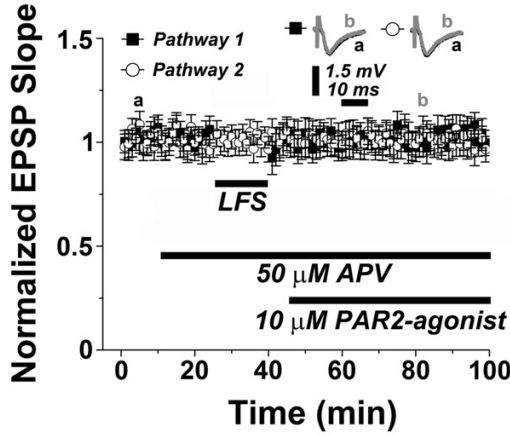

B

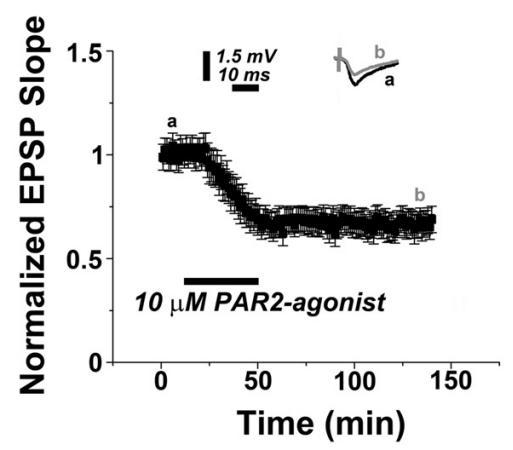

D

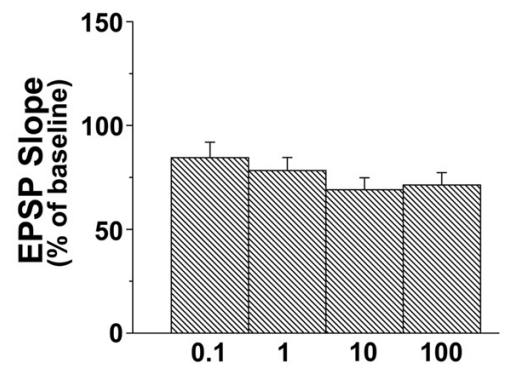

F

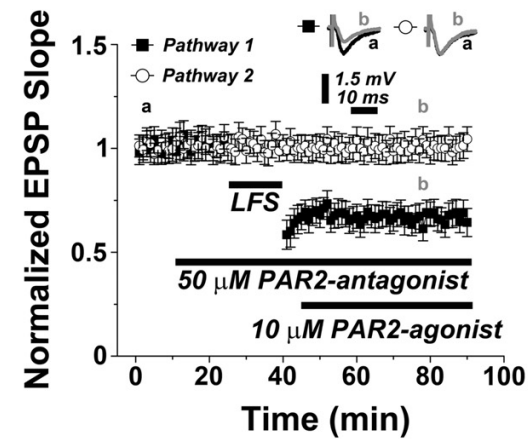

H

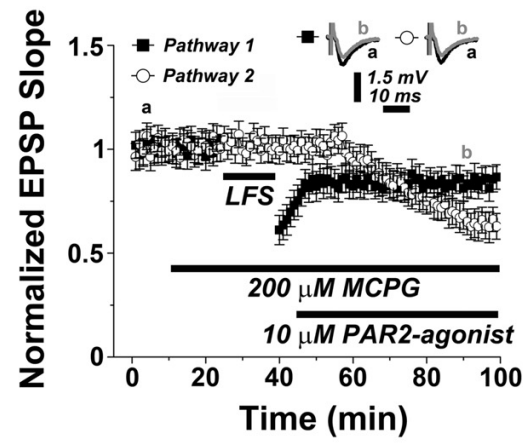

FIGURE 1 | PAR2-activation induces a depression of synaptic transmission at Schaffer collateral-CA1 synapses in the hippocampus. (A) Application of PAR2-agonist (10 $\mu \mathrm{M}$ AC55541) causes LTD. (B) Removal of the PAR2-agonist (10 $\mu \mathrm{M}$ AC55541) following induction of LTD does not affect the stability of synaptic depression. (C) In presence of a PAR2-antagonist (50 $\mu \mathrm{M}$ FSLLRY-NH 2 ) the PAR2-agonist (10 $\mu \mathrm{M}$ AC55541) is not able to induce synaptic depression.

(D) Application of PAR2-agonist (10 $\mu \mathrm{M}$ AC55541) at different concentrations results in similar levels of synaptic depression. (E) In a two pathways experimental setting, low frequency stimulation (LFS, $1 \mathrm{~Hz}, 900$ pulses) and PAR2-activation (10 $\mu \mathrm{M}$ AC55541) induce similar levels of LTD. (F) LFS-induced LTD is not blocked by the PAR2-antagonist. (G) In a two pathways experimental setting, the NMDAR-antagonist (50 $\mu$ M APV) blocks both LFS-induced LTD and PAR2-induced LTD. (H) While the group I mGluR-antagonist MCPG $(200 \mu \mathrm{M})$ partially affects LFS-LTD it does not influence PAR2-LTD. Averaged EPSP are plotted versus time. Representative traces at indicated times $(a, b)$ are shown on top of each section, $n=12$ slices for each experiment, refer to text for statistics. 


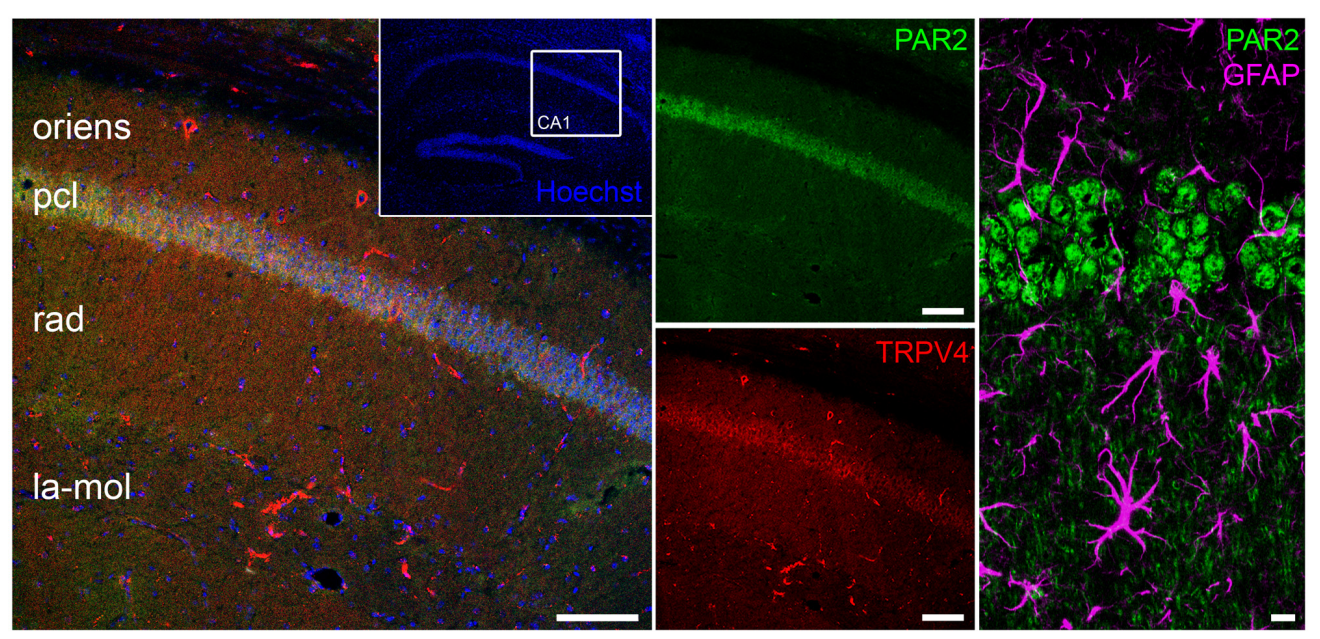

FIGURE 2 | PAR2 and TRPV4 expression in the hippocampus. Immunohistochemistry discloses the expression of PAR2 and TRPV4 in the hippocampus. A comparable expression pattern is observed: high levels of PAR2 and TRPV4 are detected in CA1 stratum pyramidale (pcl, pyramidal cell layer; oriens, stratum oriens; rad, stratum radiatum; la-mol, stratum lacunosum-moleculare). No pronounced colocalization between PAR2 and GFAP was detected. Scale bars: 100 and $10 \mu \mathrm{m}, n=9$ slices out of three animals.

To verify that pharmacological activation of PAR2 or TRPV4 induces a genuine LTD at Shaffer collateral-CA1 synapses, in a separate set of experiments we systematically assessed input/output curves and paired-pulse ratios and we did not find any significant effects on these parameters (Supplementary Figure S2). Taken together, we conclude that PAR2 mediates NMDAR-dependent LTD through the activation of TRPV4.

\section{PAR2-Induced LTD Requires Protein Kinase A}

Previous work has indicated that PAR2 acts through PKA to activate TRPV4 in inflammation and pain (Zhao et al., 2015). Hence, to provide further evidence for our major conclusion, we decided to test whether PKA is involved in mediating PAR2LTD. This hypothesis is relevant also for the known role of PKA as a canonical signaling molecule associated with hippocampal LTD (Collingridge et al., 2010; Hell, 2016; Sanderson et al., 2016). Indeed, in presence of the PKA-inhibitor KT5720 (2 $\mu \mathrm{M})$ PAR2activation failed to induce LTD (Figure 5A). This effect was specific, since pharmacological inhibition of protein kinase $\mathrm{C}$ (PKC), an additional molecule reported to be involved in LTD (Collingridge et al., 2010), had no apparent effect on PAR2-LTD (Figure 5B).

\section{DISCUSSION}

The present study addresses the role of PAR2-mediated signaling pathways in synaptic plasticity of central neurons. Based on our results we propose a model in which PAR2-activation induces (PKA-dependent) TRPV4-mediated LTD, which requires NMDAR-activity. Hence, our study is the first to report that PAR2 induces LTD at central synapses via TRPV4.
Work from recent years has identified an important role of PARs in the regulation of synaptic plasticity in the central nervous system. In this context the role of PAR1 has been extensively studied (Ben Shimon et al., 2015). It has been demonstrated that PAR1-activation induces LTP, which affects the ability of neurons to express further plasticity (Maggio et al., 2008; Stein et al., 2015). The results of the present study now demonstrate that in stark contrast to PAR1, PAR2 induces LTD (see also Gan et al., 2011). Considering that different proteases activate PAR1 and PAR2, it is interesting to hypothesize that PAR1- and PAR2-mediated synaptic plasticity could be the target of distinct protease signaling pathways, which aim at setting and modulating the threshold and direction of synaptic plasticity by balancing LTP and LTD.

This suggestion is of considerable relevance in the context of neurological diseases associated with the break-down of the blood brain barrier and/or increased brain proteases levels, e.g., brain thrombin concentrations (Chapman, 2006; Maggio et al., 2013a; Itzekson et al., 2014; Bushi et al., 2015; Deselms et al., 2016; Itsekson-Hayosh et al., 2016). Evidence has been provided that thrombin-induced PAR1-mediated LTP saturates and thus occludes the ability of neurons to express synaptic plasticity. Accordingly, it has been hypothesized that PAR1-inhibitors may improve the ability of neurons to express plasticity by preventing the pathological induction of LTP (Maggio et al., 2013b, 2014; Becker et al., 2014). However, it has been also recognized that the effects of PAR1 on synaptic plasticity can differ depending on the activating proteases and the concentration of activating ligands - a phenomenon termed 'biased agonism' (Grimsey et al., 2011). For example low concentrations of thrombin, or a specific PAR1-agonist, improve the ability of neurons to express synaptic plasticity without inducing LTP per se (Maggio et al., 2013 b 2014). Thus, while in a recent study we were not able to detect adverse effects of prolonged PAR1-inhibition on dendritic 


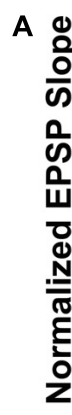

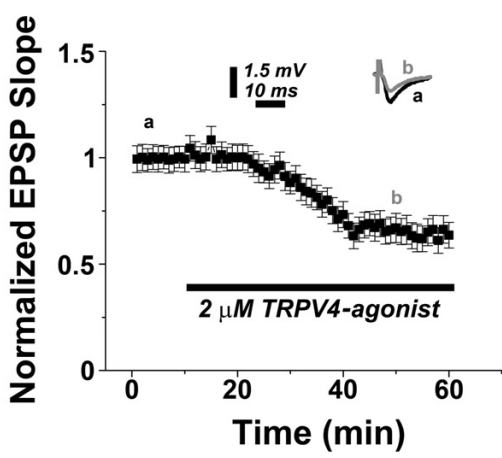

C

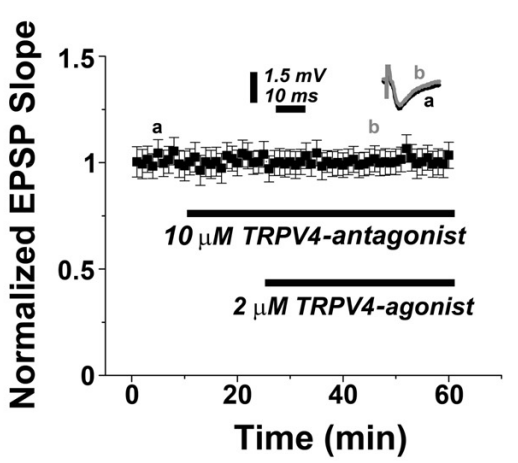

E

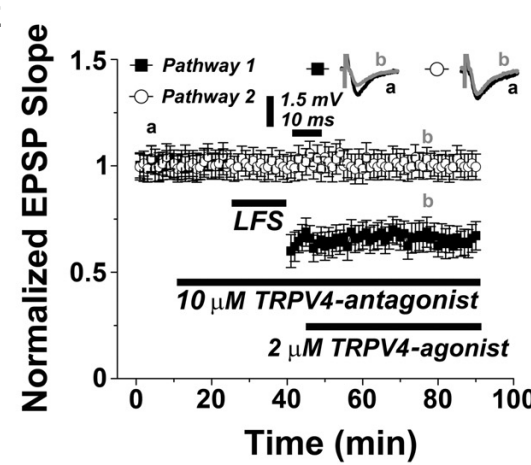

G

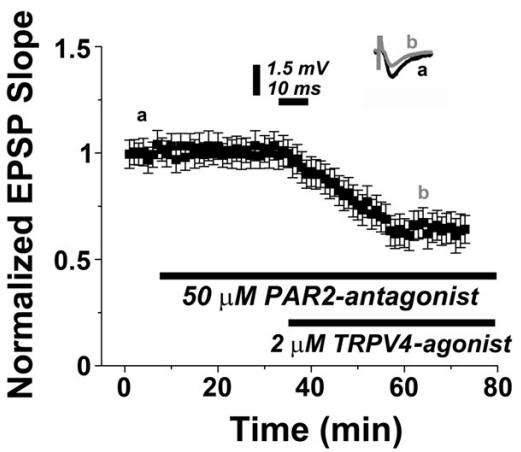

B

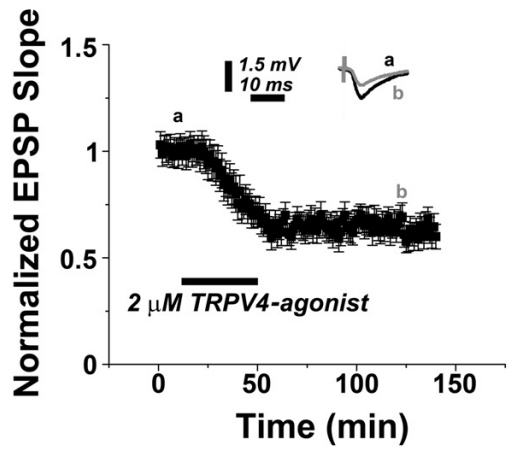

D

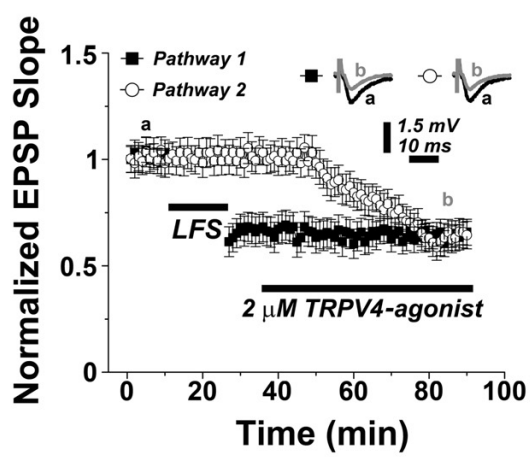

F

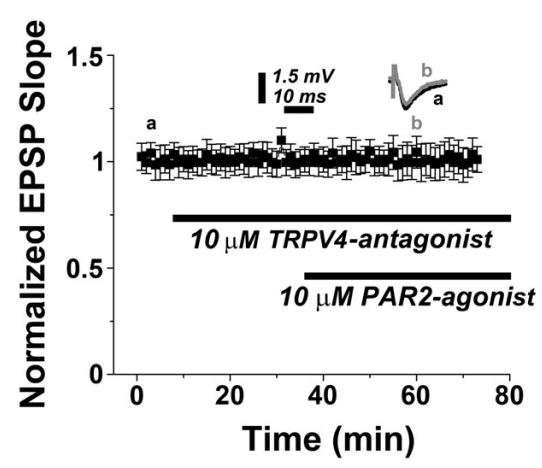

H

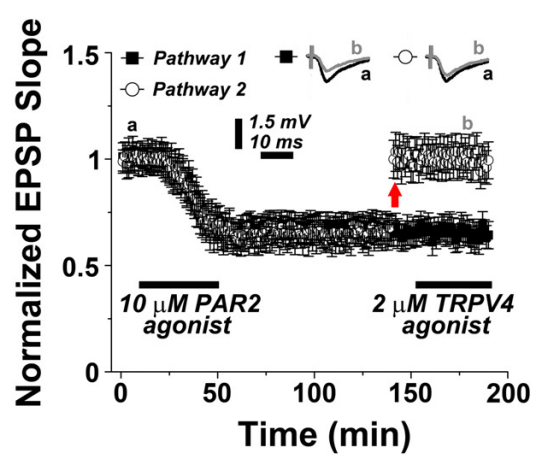

FIGURE 3 | PAR2 induces LTD through the activation of TRPV4. (A) Application of TRPV4-agonist (2 $\mu$ M RN1747) causes LTD. (B) Removal of the TRPV4-agonist ( $2 \mu \mathrm{M}$ RN1747) following induction of LTD does not affect the stability of synaptic depression. (C) In presence of the TRPV4-antagonist (10 $\mu \mathrm{M}$ RN1734) the TRPV4-agonist is not able to induce synaptic depression. (D) In a two pathways experimental setting, low frequency stimulation (LFS, $1 \mathrm{~Hz}$, 900 pulses) and TRPV4-agonist application induce similar levels of LTD. (E) LFS-induced LTD is not blocked by the TRPV4-antagonist. (F) Application of PAR2-agonist (10 $\mu \mathrm{M}$ AC55541) in presence of a TRPV4-antagonist (10 $\mu \mathrm{M}$ RN1734) blocks PAR2-induced LTD. (G) Application of TRPV4-agonist (2 $\mu \mathrm{M}$ RN1747) in presence of PAR2-antagonist (50 $\mu$ M FSLLRY-NH ${ }_{2}$ ) does not affect TRPV4-induced LTD. (H) Once PAR2-agonist mediated LTD is established, the TRPV4-agonist (2 $\mu$ M RN1747) does not further de-potentiate a second pathway at adjusted response level (upward arrow). Averaged EPSP are plotted versus time. Representative traces at indicated times $(a, b)$ are shown on top of each section, $n=12$ slices for each experiments, refer to text for statistics. 
A

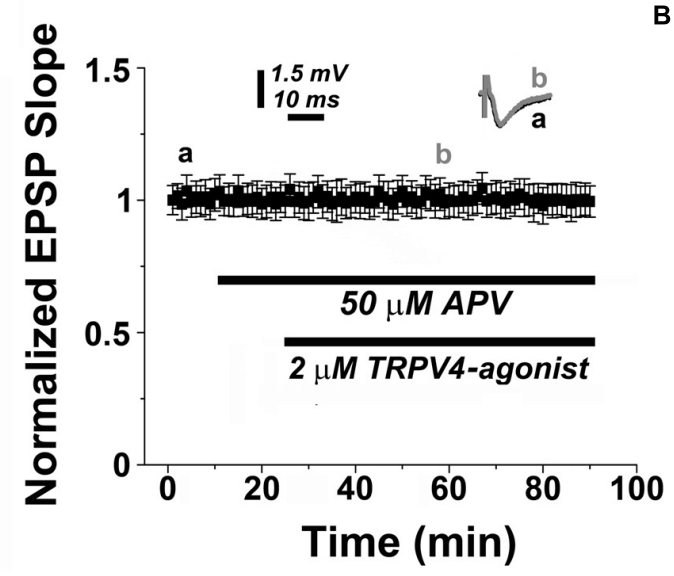

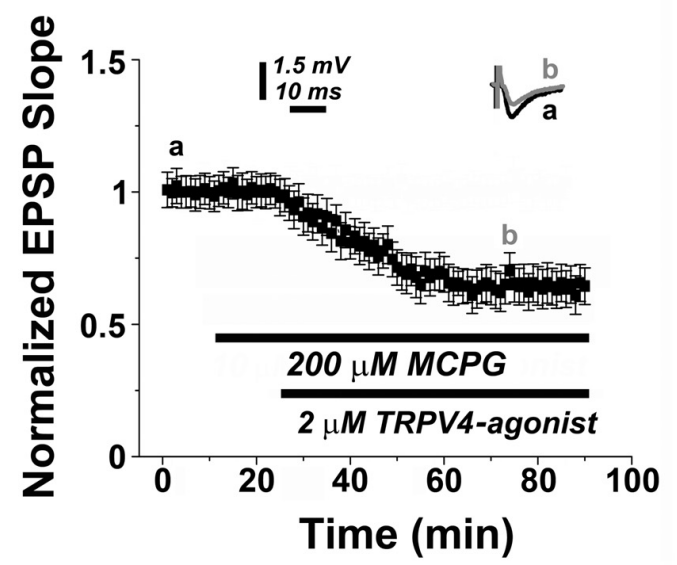

FIGURE 4 | TRPV4-mediated LTD depends on NMDAR-activity. (A) Similar to PAR2-induced LTD (c.f., Figures 1G,H), the NMDAR-antagonist (50 $\mu$ M APV) blocks TRPV4 (2 $\mu$ M RN1747)-induced LTD, while (B) application of a TRPV4-agonist (2 $\mu$ M RN1747) induces LTD in presence of the mGluR-antagonist (200 $\mu$ M MCGP). Averaged EPSP are plotted versus time. Representative traces at indicated times (a, b) are shown on top of each section.
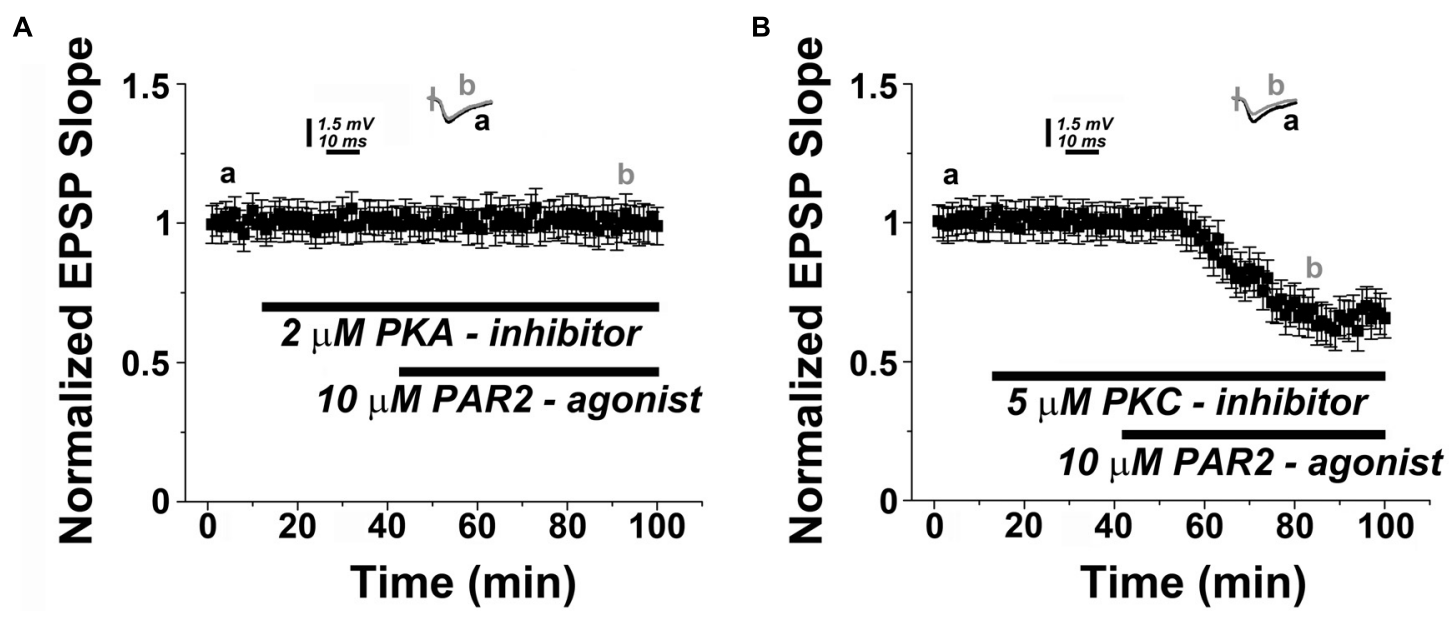

FIGURE 5 | PAR2-mediated LTD is protein kinase A (PKA)-dependent. (A) PAR2-agonist (10 $\mu$ M AC55541) in presence of a PKA-inhibitor (2 $\mu$ M KT5720) KT5720 (2 $\mu$ M) fails to induce LTD. (B) Application of a protein kinase C (PKC) inhibitor (2 $\mu$ M GF109203x) does not affect the induction of PAR2-mediated LTD. Averaged EPSP are plotted versus time. Representative traces at indicated times $(\mathrm{a}, \mathrm{b})$ are shown on top of each section.

plasticity of neurons (Schuldt et al., 2016), it is conceivable that pharmacological inhibition of PAR1 may not only exert positive effects on neural plasticity.

A similar dose-dependent effect of PAR2-activation on synaptic plasticity was not detected in the present study, as distinct concentrations of the specific PAR2-agonist reliably induced LTD. Although these results do not rule out an impact of 'biased agonism' on PAR2-mediated synaptic plasticity, it is interesting to speculate that PAR2-induced synaptic depression may counteract detrimental effects of PAR1-LTP in a 'doseindependent manner.' Such interaction between PAR1 and PAR2 may follow the rules of metaplasticity (Abraham, 2008; Hulme et al., 2013) and thus PAR2-LTD could robustly reverse or re-set the threshold of synaptic plasticity under conditions of a thrombin/PAR1-LTP mediated saturation of synaptic plasticity. Whether PAR2-agonists could prove suitable for the treatment of stroke and other diseases associated with increased brain thrombin levels or PAR1-activity is matter of current investigations. Apparently, in this context the role of distinct proteases and PARs in mediating metaplasticity, i.e., balancing neural plasticity under physiological and pathological conditions, needs to be determined.

Regardless of these considerations our work demonstrates that PAR2-LTD is mediated via TRPV4. Previous work has linked TRPV channels to the regulation of synaptic plasticity (Kauer and Gibson, 2009). It has been shown for example that TRPV1 mediates LTD of excitatory synapses on interneurons through an endocannabinoid-mGluR-dependent mechanism (Brown et al., 2013). Whether this mechanism is also mediated by PAR2 is not known. Notably, our data show that PAR2-TRPV4-mediated 
LTD of excitatory synapses on hippocampal principal neurons is not mGluR-dependent. These findings raise important questions regarding the cellular and subcellular distribution of PAR2 and distinct TRPVs, and how this distribution may affect the specific response to the exposure of distinct proteases. In this context the slow kinetics of PAR2/TRPV4mediated LTD, compared to the one observed in the LFS induced LTD, may possibly depend on a delay by which PAR2-activation promotes glutamate release from the synaptic terminals via TRPV4 (Kauer and Gibson, 2009; Hunt et al., 2012). Astrocytic PAR2 expression (Bushell et al., 2006) could play an important role in orchestrating this process. However, in the present study we were not able to detect a robust astrocytic PAR2-signal in the hippocampus. Thus additional work is required to determine the precise distribution of PAR2 and TRPV in distinct brain regions, cell types and neural compartments in order to learn more about how PAR2-signaling pathways regulate the ability of neurons to express synaptic plasticity through TRPVactivation.

While our results stand along with the work from TRPV4deficient mice, which show impaired neuronal excitability and altered social as well as depressed behavior (Shibasaki et al., 2015), the precise mechanisms how NMDARs mediate PAR2TRPV4-LTD and the functional relevance of this mechanisms for physiological and pathological brain functions is unclear. At this point we can only state that PAR2-TRPV4-mediated LTD is NMDAR- and not mGluR-dependent. Considering that a link between PAR2 and TRPV4 has already been established in the peripheral nerve system (Grant et al., 2007; Chen et al., 2011; Poole et al., 2013), where proteases that activate PAR2 sensitize TRPV-channels, which amplifies the proinflammatory and hyperalgesic actions of proteases (Noorbakhsh et al., 2006; Poole et al., 2013), our study provides first evidence that similar interactions, i.e., (PKA-dependent) PAR2-mediated TRPV4 sensitization is also relevant in mediating

\section{REFERENCES}

Abraham, W. C. (2008). Metaplasticity: tuning synapses and networks for plasticity. Nat. Rev. Neurosci. 9:387. doi: 10.1038/nrn2356

Almonte, A. G., Hamill, C. E., Chhatwal, J. P., Wingo, T. S., Barber, J. A., Lyuboslavsky, P. N., et al. (2007). Learning and memory deficits in mice lacking protease activated receptor-1. Neurobiol. Learn. Mem. 88, 295-304. doi: 10. 1016/j.nlm.2007.04.004

Almonte, A. G., Qadri, L. H., Sultan, F. A., Watson, J. A., Mount, D. J., Rumbaugh, G., et al. (2013). Protease-activated receptor-1 modulates hippocampal memory formation and synaptic plasticity. J. Neurochem. 124, 109-122. doi: 10.1111/jnc.12075

Alter, B. J., and Gereau, R. W. T. (2008). Hotheaded: TRPV1 as mediator of hippocampal synaptic plasticity. Neuron 57, 629-631. doi: 10.1016/j.neuron. 2008.02.023

Bao, Y., Hou, W., and Hua, B. (2014). Protease-activated receptor 2 signalling pathways: a role in pain processing. Expert Opin. Ther. Targets 18, 15-27. doi: 10.1517/14728222.2014.844792

Becker, D., Ikenberg, B., Schiener, S., Maggio, N., and Vlachos, A. (2014). NMDAreceptor inhibition restores Protease-Activated Receptor 1 (PAR1) mediated alterations in homeostatic synaptic plasticity of denervated mouse dentate and modulating synaptic plasticity in the central nervous system.

\section{AUTHOR CONTRIBUTIONS}

Conceived the study: NM, AV, JC. Conducted experiments and analyzed data: ES-S, AA-F, EF, MBS, ZI-H, NM. Wrote the paper: NM, AV.

\section{ACKNOWLEDGMENTS}

This study was supported by a Talpiot Medical Leadership Program grant from the Chaim Sheba Medical Center to NM and a German Israeli Foundation grant (GIF G-1317-418.13/2015) to AV and NM.

\section{SUPPLEMENTARY MATERIAL}

The Supplementary Material for this article can be found online at: http://journal.frontiersin.org/article/10.3389/fnmol. 2017.00042/full\#supplementary-material

FIGURE S1 | Specificity of PAR2- and TRPV4-mediated LTP. (A) Application of a different PAR2-agonist (10 $\mu$ M AC264613) resulted in similar levels of LTD. (B) In presence of a PAR2-antagonist (50 $\mu \mathrm{M}$ FSLLRY- $\left.\mathrm{NH}_{2}\right)$ the PAR2-agonist $(10 \mu \mathrm{M}$ AC264613) is also not able to induce synaptic depression; (C) A different TRPV4-antagonist (10 $\mu$ M RN9893) also blocked TRPV4-agonist ( $2 \mu \mathrm{M}$ RN1747) induced LTD. Averaged EPSP are plotted versus time. Representative traces at indicated times $(a, b)$ are shown on top of each section.

FIGURE S2 | Effects of PAR2- and TRPV4-agonists on Input/Output curves and paired pulse responses. Neither PAR2-agonist (10 $\mu \mathrm{M}$ AC55541) nor TRPV4-agonist (2 $\mu \mathrm{M}$ RN1747) affected presynaptic volley (A) and EPSP responses (B) when Input/Output curves were tested. (C) Paired pulses responses were not affected by PAR2-agonist (10 $\mu \mathrm{M}$ AC55541) or TRPV4-agonist (2 $\mu \mathrm{M}$ RN1747).

granule cells. Neuropharmacology 86, 212-218. doi: 10.1016/j.neuropharm. 2014.07.013

Ben Shimon, M., Lenz, M., Ikenberg, B., Becker, D., Shavit Stein, E., Chapman, J., et al. (2015). Thrombin regulation of synaptic transmission and plasticity: implications for health and disease. Front. Cell. Neurosci. 9:151. doi: 10.3389/ fncel.2015.00151

Brown, T. E., Chirila, A. M., Schrank, B. R., and Kauer, J. A. (2013). Loss of interneuron LTD and attenuated pyramidal cell LTP in Trpvl and Trpv3 KO mice. Hippocampus 23, 662-671. doi: 10.1002/hipo.22125

Bushell, T. J., Cunningham, M. R., Mcintosh, K. A., Moudio, S., and Plevin, R. (2016). Proteinase-activated receptor 2: are common functions in glial and immune cells linked to inflammation-related CNS disorders? Curr. Drug Targets 17, 1861-1870. doi: 10.2174/1389450117666151209115232

Bushell, T. J., Plevin, R., Cobb, S., and Irving, A. J. (2006). Characterization of proteinase-activated receptor 2 signalling and expression in rat hippocampal neurons and astrocytes. Neuropharmacology 50, 714-725. doi: 10.1016/j. neuropharm.2005.11.024

Bushi, D., Ben Shimon, M., Shavit Stein, E., Chapman, J., Maggio, N., and Tanne, D. (2015). Increased thrombin activity following reperfusion after ischemic stroke alters synaptic transmission in the hippocampus. J. Neurochem. 135, 1140-1148. doi: $10.1111 /$ jnc. 13372 
Chapman, J. (2006). Thrombin in inflammatory brain diseases. Autoimmun. Rev. 5, 528-531. doi: 10.1016/j.autrev.2006.02.011

Chen, Y., Yang, C., and Wang, Z. J. (2011). Proteinase-activated receptor 2 sensitizes transient receptor potential vanilloid 1 , transient receptor potential vanilloid 4, and transient receptor potential ankyrin 1 in paclitaxel-induced neuropathic pain. Neuroscience 193, 440-451. doi: 10.1016/j.neuroscience.2011. 06.085

Collingridge, G. L., Peineau, S., Howland, J. G., and Wang, Y. T. (2010). Long-term depression in the CNS. Nat. Rev. Neurosci. 11, 459-473. doi: 10.1038/nrn2867

Coughlin, S. R. (2001). Protease-activated receptors in vascular biology. J. Thromb. Haemost. 86, 298-307.

Deselms, H., Maggio, N., Rubovitch, V., Chapman, J., Schreiber, S., Tweedie, D., et al. (2016). Novel pharmaceutical treatments for minimal traumatic brain injury and evaluation of animal models and methodologies supporting their development. J. Neurosci. Methods 272, 69-76. doi: 10.1016/j.jneumeth.2016. 02.002

Edwards, J. G., Gibson, H. E., Jensen, T., Nugent, F., Walther, C., Blickenstaff, J., et al. (2012). A novel non-CB1/TRPV1 endocannabinoid-mediated mechanism depresses excitatory synapses on hippocampal CA1 interneurons. Hippocampus 22, 209-221. doi: 10.1002/hipo.20884

Fitzjohn, S., Bashir, Z., and Farrow, P. (2016). Group I mGluR induced LTD of NMDAR-synaptic transmission at the Schaffer collateral but not temperoammonic input to CA1. Curr. Neuropharmacol. 14, 435-440. doi: 10. 2174/1570159X13666150615221502

Gan, J., Greenwood, S. M., Cobb, S. R., and Bushell, T. J. (2011). Indirect modulation of neuronal excitability and synaptic transmission in the hippocampus by activation of proteinase-activated receptor-2. Br. J. Pharmacol. 163, 984-994. doi: 10.1111/j.1476-5381.2011.01293.x

Gibson, H. E., Edwards, J. G., Page, R. S., Van Hook, M. J., and Kauer, J. A. (2008). TRPV1 channels mediate long-term depression at synapses on hippocampal interneurons. Neuron 57, 746-759. doi: 10.1016/j.neuron.2007. 12.027

Gingrich, M. B., Junge, C. E., Lyuboslavsky, P., and Traynelis, S. F. (2000). Potentiation of NMDA receptor function by the serine protease thrombin J. Neurosci. 20, 4582-4595.

Grant, A. D., Cottrell, G. S., Amadesi, S., Trevisani, M., Nicoletti, P., Materazzi, S., et al. (2007). Protease-activated receptor 2 sensitizes the transient receptor potential vanilloid 4 ion channel to cause mechanical hyperalgesia in mice. J. Physiol. 578, 715-733. doi: 10.1113/jphysiol.2006.121111

Grimsey, N., Soto, A. G., and Trejo, J. (2011). Regulation of protease-activated receptor signaling by post-translational modifications. IUBMB Life 63, 403-411. doi: 10.1002/iub.442

Gunthorpe, M. J., Benham, C. D., Randall, A., and Davis, J. B. (2002). The diversity in the vanilloid (TRPV) receptor family of ion channels. Trends Pharmacol. Sci. 23, 183-191. doi: 10.1016/S0165-6147(02)01999-5

Hell, J. W. (2016). How Ca2+-permeable AMPA receptors, the kinase PKA, and the phosphatase PP2B are intertwined in synaptic LTP and LTD. Sci. Signal. 9:e2. doi: $10.1126 /$ scisignal.aaf7067

Hulme, S. R., Jones, O. D., Raymond, C. R., Sah, P., and Abraham, W. C. (2013). Mechanisms of heterosynaptic metaplasticity. Philos. Trans. R. Soc. Lond. B Biol. Sci. 369, 20130148. doi: 10.1098/rstb.2013.0148

Hunt, R. F., Hortopan, G. A., Gillespie, A., and Baraban, S. C. (2012). A novel zebrafish model of hyperthermia-induced seizures reveals a role for TRPV4 channels and NMDA-type glutamate receptors. Exp. Neurol. 237, 199-206. doi: 10.1016/j.expneurol.2012.06.013

Itsekson-Hayosh, Z., Shavit-Stein, E., Katzav, A., Rubovitch, V., Maggio, N., Chapman, J., et al. (2016). Minimal traumatic brain injury in mice: proteaseactivated receptor 1 and thrombin-related changes. J. Neurotrauma 33, 1848-1854. doi: 10.1089/neu.2015.4146

Itzekson, Z., Maggio, N., Milman, A., Shavit, E., Pick, C. G., and Chapman, J. (2014). Reversal of trauma-induced amnesia in mice by a thrombin receptor antagonist. J. Mol. Neurosci. 53, 87-95. doi: 10.1007/s12031-013-0200-8

Kauer, J. A., and Gibson, H. E. (2009). Hot flash: TRPV channels in the brain. Trends Neurosci 32, 215-224. doi: 10.1016/j.tins.2008.12.006

Lee, C. J., Mannaioni, G., Yuan, H., Woo, D. H., Gingrich, M. B., and Traynelis, S. F. (2007). Astrocytic control of synaptic NMDA receptors. J. Physiol. 581, 1057-1081. doi: 10.1113/jphysiol.2007.130377
Lohman, R. J., Jones, N. C., O’brien, T. J., and Cocks, T. M. (2009). A regulatory role for protease-activated receptor-2 in motivational learning in rats. Neurobiol. Learn. Mem. 92, 301-309. doi: 10.1016/j.nlm.2009.03.010

Luo, W., Wang, Y., and Reiser, G. (2007). Protease-activated receptors in the brain: receptor expression, activation, and functions in neurodegeneration and neuroprotection. Brain Res. Rev. 56, 331-345. doi: 10.1016/j.brainresrev.2007. 08.002

Macfarlane, S. R., Seatter, M. J., Kanke, T., Hunter, G. D., and Plevin, R. (2001). Proteinase-activated receptors. Pharmacol. Rev. 53, 245-282.

Maggio, N., Blatt, I., Vlachos, A., Tanne, D., Chapman, J., and Segal, M. (2013a). Treating seizures and epilepsy with anticoagulants? Front. Cell. Neurosci. 7:19. doi: $10.3389 /$ fncel.2013.00019

Maggio, N., Itsekson, Z., Dominissini, D., Blatt, I., Amariglio, N., Rechavi, G., et al. (2013b). Thrombin regulation of synaptic plasticity: implications for physiology and pathology. Exp. Neurol. 247, 595-604. doi: 10.1016/j.expneurol.2013.02.011

Maggio, N., Itsekson, Z., Ikenberg, B., Strehl, A., Vlachos, A., Blatt, I., et al. (2014). The anticoagulant activated protein $\mathrm{C}(\mathrm{aPC})$ promotes metaplasticity in the hippocampus through an EPCR-PAR1-S1P1 receptors dependent mechanism. Hippocampus 24, 1030-1038. doi: 10.1002/hipo.22288

Maggio, N., and Segal, M. (2007a). Striking variations in corticosteroid modulation of long-term potentiation along the septotemporal axis of the hippocampus. J. Neurosci. 27, 5757-5765.

Maggio, N., and Segal, M. (2007b). Unique regulation of long term potentiation in the rat ventral hippocampus. Hippocampus 17, 10-25. doi: 10.1002/hipo.20237

Maggio, N., and Segal, M. (2009). Differential modulation of long-term depression by acute stress in the rat dorsal and ventral hippocampus. J. Neurosci. 29, 8633-8638. doi: 10.1523/JNEUROSCI.1901-09.2009

Maggio, N., Shavit, E., Chapman, J., and Segal, M. (2008). Thrombin induces longterm potentiation of reactivity to afferent stimulation and facilitates epileptic seizures in rat hippocampal slices: toward understanding the functional consequences of cerebrovascular insults. J. Neurosci. 28, 732-736. doi: 10.1523/ JNEUROSCI.3665-07.2008

Maggio, N., and Vlachos, A. (2014). Synaptic plasticity at the interface of health and disease: new insights on the role of endoplasmic reticulum intracellular calcium stores. Neuroscience 281C, 135-146. doi: 10.1016/j.neuroscience.2014.09.041

Malenka, R. C. (2003). The long-term potential of LTP. Nat. Rev. Neurosci. 4, 923-926. doi: 10.1038/nrn1258

Nieman, M. T. (2016). Protease-activated receptors in hemostasis. Blood 128, 169-177. doi: 10.1182/blood-2015-11-636472

Noorbakhsh, F., Tsutsui, S., Vergnolle, N., Boven, L. A., Shariat, N., Vodjgani, M., et al. (2006). Proteinase-activated receptor 2 modulates neuroinflammation in experimental autoimmune encephalomyelitis and multiple sclerosis. J. Exp. Med. 203, 425-435. doi: 10.1084/jem.20052148

Oikonomopoulou, K., Hansen, K. K., Saifeddine, M., Vergnolle, N., Tea, I., Diamandis, E. P., et al. (2006). Proteinase-mediated cell signalling: targeting proteinase-activated receptors (PARs) by kallikreins and more. Biol. Chem. 387, 677-685. doi: 10.1515/bc.2006.086

Olianas, M. C., Dedoni, S., and Onali, P. (2007). Proteinase-activated receptors 1 and 2 in rat olfactory system: layer-specific regulation of multiple signaling pathways in the main olfactory bulb and induction of neurite retraction in olfactory sensory neurons. Neuroscience 146, 1289-1301. doi: 10.1016/j. neuroscience.2007.02.059

Poole, D. P., Amadesi, S., Veldhuis, N. A., Abogadie, F. C., Lieu, T., Darby, W., et al. (2013). Protease-activated receptor 2 (PAR2) protein and transient receptor potential vanilloid 4 (TRPV4) protein coupling is required for sustained inflammatory signaling. J. Biol. Chem. 288, 5790-5802. doi: 10.1074/jbc.M112. 438184

Rezaie, A. R. (2014). Protease-activated receptor signalling by coagulation proteases in endothelial cells. Thromb. Haemost. 112, 876-882. doi: 10.1160/ TH14-02-0167

Rothmeier, A. S., and Ruf, W. (2012). Protease-activated receptor 2 signaling in inflammation. Semin. Immunopathol. 34, 133-149. doi: 10.1007/s00281-0110289-1

Sanderson, J. L., Gorski, J. A., and Dell'acqua, M. L. (2016). NMDA receptordependent LTD requires transient synaptic incorporation of $\mathrm{Ca}(2)(+)$ permeable AMPARs mediated by AKAP150-anchored PKA and calcineurin. Neuron 89, 1000-1015. doi: 10.1016/j.neuron.2016.01.043 
Schuldt, G., Galanis, C., Strehl, A., Hick, M., Schiener, S., Lenz, M., et al. (2016). Inhibition of protease-activated receptor 1 does not affect dendritic homeostasis of cultured mouse dentate granule cells. Front Neuroanat 10:64. doi: 10.3389/ fnana.2016.00064

Shibasaki, K., Sugio, S., Takao, K., Yamanaka, A., Miyakawa, T., Tominaga, M., et al. (2015). TRPV4 activation at the physiological temperature is a critical determinant of neuronal excitability and behavior. Pflugers Arch. 467, 2495-2507. doi: 10.1007/s00424-015-1726-0

Stein, E. S., Itsekson-Hayosh, Z., Aronovich, A., Reisner, Y., Bushi, D., Pick, C. G., et al. (2015). Thrombin induces ischemic LTP (iLTP): implications for synaptic plasticity in the acute phase of ischemic stroke. Sci. Rep. 5:7912. doi: 10.1038/ srep07912

Tillu, D. V., Hassler, S. N., Burgos-Vega, C. C., Quinn, T. L., Sorge, R. E., Dussor, G., et al. (2015). Protease-activated receptor 2 activation is sufficient to induce the transition to a chronic pain state. Pain 156, 859-867. doi: 10.1097/j.pain. 0000000000000125

Traynelis, S. F., and Trejo, J. (2007). Protease-activated receptor signaling: new roles and regulatory mechanisms. Curr. Opin. Hematol. 14, 230-235. doi: 10. 1097/MOH.0b013e3280dce568

Tsilibary, E., Tzinia, A., Radenovic, L., Stamenkovic, V., Lebitko, T., Mucha, M., et al. (2014). Neural ECM proteases in learning and synaptic plasticity. Prog. Brain Res. 214, 135-157. doi: 10.1016/B978-0-444-63486-3.00006-2
Vance, K. M., Rogers, R. C., and Hermann, G. E. (2015). PAR1-activated astrocytes in the nucleus of the solitary tract stimulate adjacent neurons via NMDA receptors. J. Neurosci. 35, 776-785. doi: 10.1523/JNEUROSCI.3105-14.2015

Wlodarczyk, J., Mukhina, I., Kaczmarek, L., and Dityatev, A. (2011). Extracellular matrix molecules, their receptors, and secreted proteases in synaptic plasticity. Dev. Neurobiol. 71, 1040-1053. doi: 10.1002/dneu.20958

Zhao, P., Lieu, T., Barlow, N., Sostegni, S., Haerteis, S., Korbmacher, C., et al. (2015). Neutrophil elastase activates protease-activated receptor-2 (PAR2) and Transient Receptor Potential Vanilloid 4 (TRPV4) to cause inflammation and pain. J. Biol. Chem. 290, 13875-13887. doi: 10.1074/jbc.M115.642736

Conflict of Interest Statement: The authors declare that the research was conducted in the absence of any commercial or financial relationships that could be construed as a potential conflict of interest.

Copyright (c) 2017 Shavit-Stein, Artan-Furman, Feingold, Ben Shimon, ItzeksonHayosh, Chapman, Vlachos and Maggio. This is an open-access article distributed under the terms of the Creative Commons Attribution License (CC BY). The use, distribution or reproduction in other forums is permitted, provided the original author(s) or licensor are credited and that the original publication in this journal is cited, in accordance with accepted academic practice. No use, distribution or reproduction is permitted which does not comply with these terms. 